\title{
Relativismo cultural en el Consejo de Derechos Humanos de las Naciones Unidas
}

\author{
Cultural Relativism in the United \\ Nations Human Rights Council
}

ROGER LLORET BLACKBURN ${ }^{1}$

Resumen: Este artículo ${ }^{2}$ identifica los estados que son partidarios de aplicar el relativismo cultural en los derechos humanos y a la vez detalla sus argumentos, todo ello a tenor de la información que facilita el Consejo de Derechos Humanos de las Naciones Unidas. Con distintos grados de intensidad, el relativismo cultural moderno aplicado a los derechos humanos se encuentra representado por tres grupos de países: en primer lugar, un grupo de repúblicas que fundamentan sus reivindicaciones en el discurso islámico; en segundo lugar, un grupo de repúblicas que basan sus argumentos en planteamientos socialistas o comunistas; y finalmente un grupo de

\footnotetext{
1 Abogado internacional y politólogo. Graduado y Máster en Derecho por la Universidad de Barcelona (España); Máster de Derecho Internacional por la Universidad de Leiden, La Haya (Países Bajos); Licenciado en Ciencias Políticas y de la Administración, con especialidad en Relaciones Internacionales, por la Universidad Autónoma de Barcelona (España) y la Universidad de Umeå (Suecia); Posgrado en Conflictos Internacionales por la Universidad Carlos III de Madrid (España). Ha contribuido en la Organización de las Naciones Unidas: en la Oficina del Alto Comisionado para los Derechos Humanos y en el Consejo de Derechos Humanos; en el Tribunal Penal Internacional para la ex Yugoslavia; y en la Misión de Estabilización en Haití.

2 El presente artículo es una traducción del inglés realizada por el autor, siendo una versión revisada y actualizada, que incluye a Singapur y Siria, que no habían completado el Examen Periódico Universal a la fecha de la primera publicación. La versión anterior a este artículo del mismo autor fue publicada en un Working Paper por el Institut Català Internacional per la Pau en 2011, titulado "Cultural Relativism in the Universal Periodic Review of the Human Rights Council".
} 
países que sitúan una concepción multicultural del estado como el origen de su reivindicación. Junto con la división en categorías y en grados de intensidad, el presente artículo aboga por cierta variación en la clasificación de los estados partidarios del relativismo cultural con respecto a los que fueron identificados por los teóricos al final de la Guerra Fría. En consecuencia, el presente artículo, aparte de sugerir la mencionada clasificación, pone en cuestión la inclusión de ciertos estados latinoamericanos que presentan una posición basada en el respeto por la autodeterminación individual y colectiva que se configura como compatible con los estándares internacionales de los derechos humanos.

Palabras claves: Relativismo Cultural, Naciones Unidas, Consejo de Derechos Humanos, Examen Periódico Universal.

Abstract: This article ${ }^{3}$ details the states in favour of applying cultural relativism in the field of human rights, and presents their arguments based on the information facilitated by the United Nations Human Rights Council. With a different range of intensity, modern cultural relativism applied to human rights is represented by three groups of states: firstly, a group of republics that base their arguments on the Islamic discourse; secondly, a group of republics that set their claims within socialist or communist frameworks; and finally a group of states that place their multicultural conception of the state as the origin of their pretensions. Along with this division into categories and different levels of intensity, this article advocates for a certain variation in the states that favour cultural relativism with those identified by scholars at the end of the Cold War. Thus, the present article, besides suggesting the abovementioned classification, questions the inclusion of certain Latin American states that present

\footnotetext{
3 This article is a translation prepared by the author in a revised and updated version that includes Singapore and Syria that had not completed the Universal Periodic Review by the time of its original publication. The previous version of this article, of the same author, was published in English in a Working Paper by the Institut Català Internacional per la Pau, in 2011, and was entitled "Cultural Relativism in the Universal Periodic Review of the Human Rights Council".
} 
a position based on the respect for individual and collective selfdetermination that is compatible with the international standards of human rights.

Keywords: Cultural Relativism, United Nations, Human Rights Council, Universal Periodic Review.

Recibido: 15.2.2020 Aceptado: 19.6.2020

Sumario

1. Introducción

2. Universalismo y relativismo cultural

3. El Examen Periódico Universal

4. El relativismo cultural asiático

5. El relativismo cultural en el Oriente Medio

6. El relativismo cultural en América Latina y el Caribe

\section{Conclusiones}

\section{Introducción}

La Declaración Universal de los Derechos Humanos (DUDH) ratifica el derecho a la igualdad, a la libertad y a la dignidad de todos los seres humanos, con independencia de su condición, opinión o creencias. Sin embargo, desde su proclamación en el año 1948, se ha debatido e incluso disputado que los derechos humanos puedan ser realmente universales. Este interrogante se ha fundamentado, en gran medida, tanto en el papel de la cultura, como en la capacidad de esta para determinar las prioridades morales y para configurar las relaciones entre el individuo y su comunidad. 
La teoría de los derechos humanos detecta la existencia de un conflicto entre la cultura y la universalidad que se desarrolló en la Conferencia Mundial de los Derechos Humanos celebrada en 1993 en Viena. Los expertos destacaron que los estados occidentales temían que ciertos países buscaran justificar con motivaciones culturales actos contrarios a los estándares universales que imponen los derechos humanos. Por un lado, se presentaban dichos estándares del universalismo de la mano de unos estados occidentales, encabezado por los Estados Unidos de América junto a las democracias europeas, $\mathrm{y}$ se fundamentaban en la igualdad, la indivisibilidad y la universalidad de todos los derechos humanos. Por otro lado, los otros países que apelaban a la pluralidad cultural que se extienden a China, Colombia, Cuba, Indonesia, Irán, Iraq, Malasia, México, Myanmar, Pakistán, Singapur, Siria, Vietnam y Yemen (Cerna, 1994, p. 740 y Otto, 1998, p. 10), sostenían que los derechos humanos dependen del contexto en que se aplican y, por ello, de cada cultura. Asimismo, estos países partidarios del relativismo cultural argumentaban que los derechos humanos equivalen a una expresión de los valores y de los intereses de occidente y, por tanto, constituyen una interferencia en sus asuntos internos.

Desde 2008, todos los países del mundo ven cómo sus registros en el ámbito de los derechos humanos son evaluados en un mismo proceso, en el marco del Consejo de Derechos Humanos (CDH) de la Organización de las Naciones Unidas (ONU), piedra angular de la anterior reforma de la Organización. El Examen Periódico Universal (EPU) ofrece la posibilidad de contrastar los argumentos del relativismo cultural y, asimismo, determinar concretamente los actores que amparan este relativismo. Por ello, el presente estudio ha tratado de verificar si tal fractura teórica y jurídica fundamentada en la cultura es o no aplicable al primer ciclo del EPU y, en su caso, establecer los actores y sus argumentos. 


\section{Universalismo y relativismo cultural}

Como hemos señalado, los autores en la materia de los derechos humanos indicaron la existencia de un conflicto relacionado con la diversidad cultural, que se manifestó claramente en la preparación y en la ejecución de la Conferencia Mundial de Derechos Humanos celebrada en 1993 en Viena, así como en la IV Conferencia Mundial de la Mujer, celebrada en Pekín, y en otras conferencias preparatorias para la Conferencia Mundial de Viena (Cerna, 1994 y Otto, 1998).

En la Declaración resultante de las reuniones preparatorias de la región asiática, celebradas en Bangkok en 1993, se establecía que "mientras que los derechos humanos tienen una naturaleza universal, deben ser considerados en el contexto de un proceso internacional dinámico y en una evolución de un establecimiento de unas normas que tome en consideración la importancia de las particularidades nacionales y regionales y de los diversos antecedentes históricos, culturales y religiosos" (Declaración de Bangkok, pár. 8). Dichas declaraciones, junto con unas formulaciones que establecían "la necesidad de evitar la aplicación de un doble estándar” (Declaración de Bangkok, Preámbulo y pár. 7), y el rechazo a "la imposición de valores incompatibles" (Declaración de Bangkok, Preámbulo) se posicionaron como un reflejo de la controversia y del desacuerdo entre delegaciones. Tras unas disputas similares en otras reuniones preparatorias regionales, como en Túnez en noviembre de 1992 y en San José en febrero de 1993, en la Conferencia Mundial de Viena el término "universalidad" se había convertido en un símbolo de la confrontación entre las delegaciones. Los estados occidentales temían la erosión de la universalidad de los derechos humanos y, por ello, presionaban a favor del reconocimiento del término "universalidad" y, a la vez, rechazaban que los derechos humanos puedan tener distinta aplicación en distintos países (Cerna, 1994, pp. 742-744, y Otto, 1998, pp. 8-13).

La Declaración y el Programa de Acción de Viena evidenció la divergencia con el uso repetitivo del término "universalidad", que se 
puso de manifiesto en fragmentos que anuncian "el respeto universal por [...] todos los derechos humanos” (A/CONF.157/23, párs. 1, 6 y 58), reafirmando "la importancia de asegurar la universalidad, la objetividad y evitar la selectividad” (A/CONF.157/23, pár. 32), aunque a la vez se declara la necesidad de tener en consideración "las particularidades nacionales y regionales y los distintos antecedentes históricos, culturales y religiosos" (A/CONF.157/23, pár. 5). En concreto, se señalaba que la acción continuada para poner en cuestión la universalidad de los derechos humanos provenía de China, Colombia, Cuba, Indonesia, Irán, Iraq, Malasia, México, Myanmar, Pakistán, Singapur, Siria, Vietnam y Yemen (Cerna, 1994, p. 740 y Otto, 1998, p. 10). Por ello, el presente estudio ha tenido en especial consideración dichos países, aunque también ha analizado las contribuciones y los exámenes de todos los estados que han realizado el primer ciclo del EPU, sin haber podido identificar los argumentos del relativismo cultural en ninguna otra aportación o examen de otros países al margen de los aquí presentados.

Por un lado, el universalismo se define con la defensa de que la cultura es irrelevante para la validez de las normas morales y reafirma la universalidad, la indivisibilidad, la igualdad y la interdependencia de todos los derechos humanos (Donnelly, 1984, p. 400 y 2007, p. 281, Otto, 1998, p. 8). Como tal, la postura universalista niega completamente que los estándares universales de los derechos humanos puedan ser subordinados a una cultura concreta. Esta postura, que procede principalmente de estados occidentales, defiende que diluir en lo más mínimo el universalismo comportaría la aceptación de los abusos de los gobiernos tiránicos. A pesar de ello, algunos sectores teóricos expresaron preocupación porque esta postura implicaría la negación de la autonomía y la autodeterminación nacional y subnacional y, por ello, sería proclive al imperialismo. Estas posiciones señalaban la capacidad potencial para anular la diversidad cultural de los derechos humanos cuando son interpretados bajo un prisma de radicalidad universalista (Donnelly 1984, p. 400 y 2007, p. 281). Dicho argumento se entiende 
tácitamente reconocido con la advertencia de la Sociedad Antropológica Americana a la Comisión de Derechos Humanos de la ONU durante la redacción de la DUDH, que alertaba del peligro de eliminar la diversidad cultural y, más aún, considerando el registro de Occidente de atribuir inferioridad cultural a los pueblos no europeos. De acuerdo con la Anthropological Society, era fundamental que los derechos humanos tuvieran en cuenta el individuo como miembro del grupo social del que forma parte (Statement on Human Rights, 1947, p. 539, Otto, 1998, p. 3).

Por otro lado, el relativismo cultural surgió de China, Colombia, Cuba, Indonesia, Irán, Iraq, Malasia, México, Myanmar, Pakistán, Singapur, Siria, Vietnam y Yemen (Cerna, 1994, p. 740 y Otto, 1998, p. 10) y un intento de redefinir el contenido de los derechos humanos que colisiona con los estados occidentales. Según Cerna, Otto y Donnelly, estos países consideran la definición de los derechos humanos como parte del patrimonio ideológico de la civilización occidental y sostienen que los principios consagrados en la DUDH reflejan los valores occidentales y no los suyos. Además, estos estados rechazan que Occidente interfiera en sus asuntos internos con la imposición de su definición de los derechos humanos y, con ello, les dificulte el crecimiento económico y debiliten su competitividad. Por ello, estos países defienden que por sus diferencias sociales y culturales no se les debe someter a los mismos criterios. Consecuentemente, el relativismo cultural defiende unas posturas alternativas a una supuesta verdad universal, que se fundamenta en unas tradiciones culturales no europeas, a la vez que rechaza el paradigma de los derechos humanos por considerarlo opresivo para los estados en vías de desarrollo y con unas culturas diferentes. Con todo, el relativismo cultural sostiene que ciertas variaciones culturales no pueden ser criticadas legítimamente por foráneos y se fundamenta en los conceptos de la autonomía y la autodeterminación de la comunidad (Cerna, 1994, p. 740, Donnelly, 1984, p. 400 y 2007, p. 293). 


\section{El Examen Periódico Universal}

El Consejo de Derechos Humanos $(\mathrm{CDH})$ se estableció en abril de 2006 por medio de una resolución de la Asamblea General (A/RES/60/251). A la vez que reafirmaba y enfatizaba los propósitos y los principios de la Declaración y Programa de Acción de Viena (A/CONF.157/23), esta resolución definía la estructura y las funciones del $\mathrm{CDH}$ para proteger y promover los derechos humanos $\mathrm{y}$, consecuentemente, los principios y los propósitos de la ONU. El CDH substituyó a la desacreditada Comisión de Derechos Humanos y representaba un componente clave de la reforma de la ONU. A diferencia de la precedente Comisión de Derechos Humanos, que dependía del Consejo Económico y Social (ECOSOC 1996/31), el CDH es un órgano dependiente de la Asamblea General de la ONU. El CDH, además se distingue por trabajar juntamente con los Estados miembros, los observadores, las organizaciones no gubernamentales, las organizaciones regionales, las instituciones nacionales y con la sociedad civil. De acuerdo con la resolución, la función del CDH es la de promover y proteger los derechos humanos, a través de la asistencia a los Estados miembros, y de una manera universal, imparcial y justa, que se base en la cooperación y en el diálogo $(\mathrm{CDH}$ 8/PRST/1, Schrijver, 2007, p. 809-813).

La capacidad que distingue al $\mathrm{CDH}$ es la de emprender "un examen periódico universal, basado en una información objetiva y fidedigna, sobre el cumplimiento por cada Estado de sus obligaciones y sus compromisos en la materia de derechos humanos, de una forma que garantice la universalidad del examen y la igualdad de trato respecto de todos los Estados” (A/RES/60/251, pár. 5). Los Estados miembros deben "cooperar plenamente con el Consejo y estarán sujetos al mecanismo del examen periódico universal durante el período en que sean miembros" (A/RES/60/251, pár. 9). El EPU es “un mecanismo cooperativo, basado en un diálogo interactivo, con la participación plena del país de que se trate y teniendo en 
consideración sus necesidades en el fomento de la capacidad" (A/RES/60/251, pár. 5).

La base general de la evaluación del EPU es la Carta de las Naciones Unidas, la DUDH, así como los tratados de derechos humanos ratificados por el estado evaluado. Cabe destacar que los principios del EPU son la promoción de la universalidad, la interdependencia, la indivisibilidad y la interrelación de "todos" los derechos humanos. La resolución establece que el desarrollo del EPU debe producirse de manera objetiva y transparente, siendo regido por criterios no selectivos y de forma constructiva y, a la vez, evitando la confrontación y la politización. Además, el EPU debe complementar y no duplicar la tarea de otros órganos de los derechos humanos y debe tener en consideración el nivel de desarrollo y las condiciones específicas del país sometido a examen. En definitiva, el objetivo del EPU es mejorar la situación de los derechos humanos en el terreno y la asistencia a los Estados miembros en su promoción y su protección (CDH 5/1).

Las contribuciones al EPU deben tener correspondencia con dichas directivas fijadas en la resolución. Los tres documentos que forman la base del EPU son: un "Informe Nacional” preparado por el estado en cuestión; una "Recopilación de la ONU” formulada por la Oficina del Alto Comisionado por los Derechos Humanos (OACDH) que expone la información contenida en la documentación de la ONU, junto con los informes de los órganos de tratados y de los procedimientos especiales de relatores de la OACDH; y finalmente, un "Resumen de los Interlocutores del EPU", que incluyen actores de la sociedad civil y de las organizaciones no gubernamentales (CDH 5/1, pár. 15). Este resumen se encuentra compilado por la OACDH y busca complementar y dar contraste a las otras dos aportaciones.

La investigación que fundamenta este análisis consideró de forma preeminente el "Informe Nacional" de todos los países en el 
primer ciclo del EPU, a modo de fuente directa de opinio juris ${ }^{4}$ del Estado sobre la cuestión de los derechos humanos y, concretamente, en lo referente al relativismo cultural. Asimismo, la investigación analizó todas las contribuciones complementarias -la Recopilación de la ONU y el Resumen de los Interlocutores- de todos los estados, así como el "diálogo interactivo" en todas las sesiones del CDH y la documentación resultante del EPU.

\section{El relativismo cultural asiático}

La República Popular China, en su Informe Nacional para el EPU, declara que "respeta el principio de la universalidad de los derechos humanos [y que la] comunidad internacional debería respetar el principio de indivisibilidad de los derechos humanos y atribuir igual importancia a los derechos civiles y políticos y a los derechos económicos, sociales y culturales, así como al derecho al desarrollo" (A/HRC/WG.6/4/CHN/1, pár. 6). A continuación añade que "habida cuenta de las diferencias existentes en los sistemas políticos, en los niveles de desarrollo y en los antecedentes históricos y culturales, es natural que los países tengan diferentes opiniones sobre la cuestión de los derechos humanos [y por ello] es importante que los países, en su esfuerzo común por promover y proteger los derechos humanos, instauren un diálogo y una cooperación basados en la igualdad y el respeto mutuo" (A/HRC/WG.6/4/CHN/1, pár. 6). El Informe también explica que "en 1949, se creó la República Popular China, y el pueblo chino ganó la independencia nacional y la liberación [y con ello] el pueblo chino se ha convertido en el verdadero dueño del país, y se ha establecido un sistema social y político para la promoción y

\footnotetext{
${ }^{4}$ Reconocido como "prueba de una práctica generalmente aceptada como derecho" en el Artículo 38 (b) del Estatuto de la Corte Internacional de Justicia, y desarrollado en la jurisprudencia en: Reino Unido v. Noruega, 18 diciembre de 1951; en Portugal v. India, 12 de abril de 1960; República Federal de Alemania v. Dinamarca/Países Bajos, 20 de febrero de 1969; Nicaragua v. Estados Unidos de América, 27 de junio de 1986, entre otros.
} 
protección de los derechos humanos" (A/HRC/WG.6/4/CHN/1, pár. $3)$.

En contraposición, el Resumen de los Interlocutores (A/HRC/WG.6/4/CHN/3) y la Recopilación de la ONU (A/HRC/WG.6/4/CHN/2) mantienen un tono crítico con la situación de los derechos humanos en la China, aunque también reconocen mejorías. Expresan, inter alia, preocupación por los informes de los órganos de tratados sobre la práctica de torturas, las penas de muerte, las condiciones de detención, la falta de independencia de la judicatura, la falta de libertad religiosa y de opinión, las condiciones laborales, la sanidad maternal e infantil, la censura y las desigualdades, así como la discriminación hacia las minorías, los refugiados y los inmigrantes. Además, en el diálogo interactivo del EPU de China (A/HRC/11/25*), se pueden distinguir dos posiciones diferenciadas. Por un lado, los estados occidentales que, a pesar de que en su mayoría reconocen progreso en los derechos humanos en China, formulan unas recomendaciones muy claras y en la línea de la documentación complementaria del EPU. Por otro lado, se detecta a otro grupo de estados que destaca y elogia los esfuerzos de la China en la materia de los derechos humanos y limita sus demandas invitando a la continuación y a la difusión de sus prácticas. Esta postura se encuentra representada por los integrantes de la lista especificada de estados partidarios del relativismo cultural, incluyendo Singapur, México, Cuba, Vietnam, Irán, Indonesia, Pakistán, Colombia y Malasia. Podemos notar, asimismo, que esta postura tiene el apoyo de un grupo substancial de países que podrían ser beneficiarios de las relaciones comerciales con China. ${ }^{5} \mathrm{La}$ fractura también se manifiesta claramente en las recomendaciones, la mayoría de las cuales son introducidas con la expresión "continuar avanzando” para los países conformistas, mientras que las

\footnotetext{
${ }^{5}$ Filipinas, Argelia, la Federación Rusa, Egipto, Bután, Libia, Sri Lanka, Arabia Saudita, Uzbekistán, Sudán, Ghana, Mozambique, Angola, Marruecos, Omán, Emiratos Árabes Unidos, Nicaragua, India, Yemen, Jordania, Baréin, Benín, Malí, Gabón, Palestina, Qatar, Venezuela, Senegal y Tailandia.
} 
recomendaciones de los países occidentales constituyen un conjunto de peticiones mucho más claras, aunque en la mayoría de los casos China las rechaza o afirma que ya se encuentran en aplicación.

El Informe Nacional de la República Socialista de Vietnam hace referencia a una "historia de lucha por la independencia nacional y la libertad, [en la que] el pueblo vietnamita siempre se ha aferrado a los valores sagrados de los derechos humanos, en particular el derecho a la libre determinación, la libertad de decidir el destino propio y el derecho a vivir con dignidad” (A/HRC/WG.6/5/VNM/1, pár. 7). A continuación, el Informe declara que los "derechos humanos más fundamentales son el derecho a vivir con independencia y libertad, el derecho a la libre determinación y el derecho a votar y a ser elegido" (A/HRC/WG.6/5/VNM/1, pár. 17). Además, el Informe Nacional de Vietnam menciona como logros y desafíos cinco "enseñanzas", la primera y la segunda de las cuales consisten en "un desarrollo nacional centrado en el pueblo [en el que] los derechos humanos son indisociables de la independencia y la soberanía nacionales [ya que la] independencia nacional es la condición y la base de la protección de los derechos humanos" (A/HRC/WG.6/5/VNM/1, párs. 57-60). Haciendo énfasis en un pasado colonial y de pueblo esclavizado, las autoridades presentan su tercera lección afirmando que a pesar de "haber sufrido muchas guerras de agresión, que suponen la violación más grave de los derechos humanos, Vietnam es plenamente consciente de que los derechos humanos revisten a un tiempo carácter universal, pues reflejan la aspiración común de la humanidad [...] y un carácter particular, propio de cada sociedad y comunidad" (A/HRC/WG.6/5/VNM/1, pár. 61). En esta tercera lección, denominada "armonización de los valores universales de los derechos humanos y las particularidades de la nación, promoción de la cooperación internacional y el diálogo en relación con los derechos humanos" (A/HRC/WG.6/5/VNM/1, párs. 61-64), también se declara que "respetando la universalidad de los derechos humanos, Vietnam ya es parte en casi todos los tratados internacionales esenciales de derechos humanos y otros tratados internacionales de esa esfera, y 
cumple a conciencia sus obligaciones" (A/HRC/WG.6/5/VNM/1, pár. 63). La cuarta lección del Vietnam se fundamenta en el "mantenimiento de la estabilidad política, promoción del desarrollo económico junto con la garantía de la seguridad social" (A/HRC/WG.6/5/VNM/1, párs. 65-66), mientras que la quinta $\mathrm{y}$ última hace referencia a "potenciar el conocimiento de la población y su capacidad para disfrutar los derechos humanos" (A/HRC/WG.6/ 5/VNM/1, párs. 67-70).

En contraposición y de un modo similar a la China, la Recopilación de la ONU (A/HRC/WG.6/5/VNM/2) y el Resumen de los Interlocutores (A/HRC/WG.6/5/VNM/3) del EPU de Vietnam expresan preocupación por las prácticas de tortura y de la aplicación de la pena de muerte, así como con la falta de libertad religiosa y de los incumplimientos en los derechos laborales y de unas condiciones adecuadas, con una mayor incidencia entre las minorías y los pueblos indígenas. Una vez más, en el debate interactivo y en las recomendaciones se pueden distinguir los dos bloques. Mientras que los países en vías de desarrollo elogian las prácticas del Vietnam en la materia de los derechos humanos y le animan a "continuar" sus prácticas en las recomendaciones de las conclusiones, los estados occidentales, junto con estados de la Europa del Este, estados bálticos y latinoamericanos ${ }^{6}$ hacen unas recomendaciones muy claras y reclaman la adopción de unas medidas correctoras concretas.

El Informe Nacional de la República de la Unión de Myanmar (A/HRC/WG.6/10/MMR/1 y A/HRC/WG.6/10/MMR/1/Corr.1) no hace referencias directas a las opiniones del país sobre la universalidad de los derechos humanos. La Recopilación de la ONU refleja preocupación respecto a los informes de los órganos de la Convención sobre la Eliminación de todas las formas de Discriminación contra la Mujer (CEDM) y especialmente "por la persistencia de unas normas, unas prácticas y unas tradiciones

${ }^{6}$ Incluyendo a Eslovaquia, Eslovenia, Hungría, República Checa, Polonia, Letonia, Chile y Argentina. 
culturales adversas en cuanto a los roles de la mujer y el hombre en todas las esferas de la vida, especialmente en algunos grupos étnicos" (A/HRC/WG.6/10/MMR/2 pár. 22). También cabe destacar que los Interlocutores denuncian Myanmar por sus "violaciones de los derechos humanos en masa, incluidos crímenes de lesa humanidad y crímenes de guerra cometidos en las últimas décadas" (A/HRC/ WG.6/10/MMR/3 pár. 6), en una situación en la que "no había prácticamente ningún marco normativo nacional para la protección de los derechos humanos como el que impone un estado de derecho" (A/HRC/WG.6/10/MMR/3 pár. 7). No obstante, Myanmar, en su Informe, afirma que ha realizado unos pasos positivos hacia la democratización y en beneficio de los derechos humanos, tanto a escala nacional como internacional, con unas referencias frecuentes a su situación poscolonial, así como a unas medidas para garantizar la libertad religiosa, el desarrollo y los derechos a la sanidad y a la educación. En el proceso interactivo de Myanmar se expresó claramente una dicotomía similar a la que se expuso en los EPU de la China y el Vietnam, con dos facciones establecidas y claramente distinguibles. Lo más destacado es que esta vez las facciones también quedan plasmadas en 13 páginas de recomendaciones, de las cuales podemos diferenciar aquellas que exhortan a mantener las prácticas del país, en contra de aquellas que las condenan y que formulan unas peticiones relacionadas con los graves problemas expuestos (A/HRC/WG.6/10/L.7).

Por lo que refiere a la República Islámica de Irán, su Informe Nacional hace referencia a una iniciativa internacional para fomentar la diversidad cultural y los derechos humanos mediante el "patrocinio de una resolución sobre los derechos humanos y la diversidad cultural en la Asamblea General de las Naciones Unidas" (A/HRC/WG.6/ 7/IRN/1, pár. 118). Además, menciona que organizó una "Reunión Ministerial del Movimiento de los Países No Alineados [MNOAL] sobre derechos humanos y diversidad cultural celebrada en Teherán en septiembre de 2007" (A/HRC/WG.6/7/IRN/1, pár. 118). Esta reunión condujo a la "creación del Centro de Derechos Humanos y 
Diversidad Cultural del MNOAL, en Teherán por los Ministros de Relaciones Exteriores del Movimiento" (A/HRC/WG.6/7/IRN/1, pár. 118). Además, dicha reunión propició el establecimiento del Centro de Derechos Humanos y Diversidad Cultural del MNOAL en Teherán, en mayo de 2008, "con el mandato principal de desarrollar y mejorar una comprensión común en la materia de los derechos humanos y la diversidad cultural entre los Estados miembros del MNOAL y entre estos y los demás miembros de la comunidad internacional" (A/HRC/ WG.6/7/IRN/1, pár. 118).

El Informe Nacional también declara que con "el triunfo de la Revolución Islámica y la salida del Irán del bloque de países occidentales, la situación de los derechos humanos en el Irán ha sido utilizada sistemáticamente como una herramienta política para ejercer presión y para promover unas determinadas intenciones políticas ocultas de algunos países occidentales específicos" (A/HRC/WG.6/7/IRN/1, pár. 128). Asimismo, afirma que de "conformidad con las disposiciones de la Declaración y el Programa de Acción de Viena aprobados en 1993 por la Conferencia Mundial de Derechos Humanos, al aplicar las normas de los derechos humanos se deben tener en cuenta las particularidades históricas, culturales y religiosas de los diferentes países y regiones" (A/HRC/WG.6/7/IRN/1, pár. 129), sin ninguna mención a la parte de la Declaración de Viena en la que se refería al universalismo.

Finalmente el Informe Nacional concluye que al "igual que otros países islámicos, el Irán ha tropezado con algunas dificultades en la puesta en práctica de algunas normas internacionales de los derechos humanos [y que la] comunidad internacional debe comprender debidamente que, habida cuenta de las estructuras jurídicas nacionales basadas en los principios del islam, la adhesión de sus autoridades a esos principios y las exigencias genuinas de la población, la República Islámica de Irán se considera obligada a respetar las leyes de la sharia islámica” (A/HRC/WG.6/7/IRN/1, pár. 130). Las autoridades añaden que en "consecuencia, es necesario que, sobre la base del principio del respeto de la diversidad cultural y 
evitando al mismo tiempo las presiones políticas y culturales, se tenga en cuenta a ese respecto que cualquier cambio o ajuste introducido en esas leyes debe surgir como resultado de la dinámica del diálogo nacional sostenido entre nuestras propias autoridades y la sociedad civil en el contexto de los principios islámicos [y que las] presiones o exigencias de otros países para que se acepten y se adopten determinadas normas occidentales de los derechos humanos tendrán en la práctica repercusiones negativas en la promoción de los derechos humanos" (A/HRC/WG.6/7/IRN/1, pár. 130).

Además, las declaraciones anteriores del Informe Nacional también fueron reproducidas por el Irán en su controvertido diálogo interactivo, en el cual se presentaba afirmando que "su situación en la materia de los derechos humanos había sido utilizada sistemáticamente por algunos países occidentales para ejercer presión e impulsar sus motivaciones políticas ocultas" (A/HRC/14/12, pár. 9). Después, haciendo énfasis en su papel en las iniciativas internacionales del relativismo cultural, la delegación iraní expresó la intención de aplicar "un enfoque interactivo y cooperativo, así como para evitar los enfrentamientos, la dualidad de criterios y la politización" (A/HRC/14/12, pár. 13). La delegación iraní concluyó su aportación afirmando "el valor de la cultura y de la historia como fuente de conocimientos en la materia de los derechos humanos, y señaló que el modo de vida liberal de Occidente no era la única opción posible [y que la] experiencia del Irán estaba arraigada en su cultura y en la fe de millones de musulmanes que respetaban plenamente la vida de las comunidades occidentales, como se reflejaba en la Constitución, que se había formulado sobre la base de modelos occidentales, pero aplicados a la racionalidad islámica" (A/HRC/14/12, pár. 89).

Cabe mencionar que los documentos complementarios contienen una información que contrasta con la aportada por Irán. La Recopilación de la ONU señala que "el Secretario General observó que la República Islámica de Irán mantenía la práctica de formular reservas generales en el momento de la firma o ratificación de 
tratados" (A/HRC/WG.6/7/IRN/2, pár. 1). También cita la aplicación generalizada de la pena de muerte y las ejecuciones públicas y, especialmente, de ejecuciones, detenciones y torturas de jóvenes ${ }^{7}$, a la vez que expresa unas fuertes preocupaciones por las libertades personales y colectivas, junto con la representación y la participación de todos los ciudadanos. En los documentos complementarios también destaca la preocupación hacia la violencia contra las mujeres, que según los informes es generalizada y, además, siguen un patrón institucionalizado, como en la imposición de un código de vestir, en su acceso a la justicia y a través de la imposición de leyes como la diyah, en las prácticas de tráfico como la siqeh, en situaciones de conversión forzada en el matrimonio y en el no reconocimiento de hijos/as de padre extranjero. También detalla la preocupación por los casos de violencia y de trabajos forzados de menores, que tienen una edad de responsabilidad penal de 14 años en el caso de los niños y de 8 años en el caso de las niñas. Por contra, las autoridades declaran que "en los últimos 30 años el Irán ha llevado a cabo de manera incesante actividades bien definidas y basadas en los principios con el fin de mejorar la condición de la mujer en las esferas educativa, política y cultural” (A/HRC/WG.6/7/IRN/1, pár. 98). Según las autoridades es por eso que se ha establecido un Consejo Cultural de la Mujer, vinculado al Alto Consejo de la Revolución Cultural, con varios comités de expertos encargados de formular políticas relativas a los asuntos culturales y sociales de la mujer y de garantizar la coordinación entre los organismos competentes (A/HRC/WG.6/7/IRN/1, pár. 103).

En cuanto a la República Islámica de Pakistán, la única referencia en su Informe Nacional se encuentra en las políticas educativas promovidas por el gobierno, sobre las cuales declara que "ha hecho un esfuerzo para incluir en el nuevo plan de estudios nacional los principios de los derechos humanos, defendiéndose la diversidad y la

7. De modo similar, el Parlamento aprobó la Convención contra la Tortura en el año 2002 pero el Consejo de Guardianes la rechazó "porque se percibieron contradicciones con los principios islámicos". 
diferencia junto a los derechos universales" (A/HRC/WG.6/2/PAK/1, pár. 74). Por otro lado, la Recopilación de la ONU afirma que el CEDM "expresó su profunda preocupación por la existencia generalizada de las actitudes patriarcales y unos estereotipos tradicionales $\mathrm{y}$ culturales profundamente arraigados respecto a las funciones y a las responsabilidades de la mujer y el hombre en la familia, en el lugar de trabajo y en la sociedad" (A/HRC/WG.6/2/PAK/2, pár. 8). Los Interlocutores, de manera parecida, comentan que "las mujeres padecían un trato mucho peor que los hombres en los tribunales, ya que tenían que enfrentarse a unos enormes prejuicios culturales [los cuales también eran] un factor agravante en los casos de homicidios en nombre del honor, ya que al parecer los jueces adoptaban a menudo una actitud indulgente hacia el autor, especialmente si era miembro de la familia de la mujer" (A/HRC/WG.6/2/PAK/3, pár. 7). Aparte de las prácticas tradicionales de castigo violento, como en el caso de la qisas, la zina y el hudood, los Interlocutores denuncian "gravísimos defectos de la propia legislación, de la administración de justicia y de los servicios de policía, así como la corrupción crónica y los prejuicios culturales que afectaban a las mujeres y a las minorías religiosas [junto con la aplicación de una] pena de muerte [...] discriminatoria e injusta, que admitía una alta probabilidad de errores en la justicia” (A/HRC/WG.6/2/PAK/3, pár. 9). En esta línea, las dos repúblicas islámicas muestran una práctica manifiesta de quebranto sistemático de la igualdad jurídica entre géneros y con la tolerancia hacia unas prácticas tradicionales que son radicalmente opuestas a los estándares de los derechos humanos.

Por lo que concierne a la República de Indonesia, en su Informe Nacional las autoridades declaran que el "Plan Nacional de Acción sobre los Derechos Humanos incluye unas medidas concretas que ha de adoptar el Gobierno a lo largo de un período de cinco años para promover y proteger los derechos humanos, de conformidad con los valores culturales, religiosos y tradicionales y sin discriminación por motivos de la raza, la religión, la etnia o facción" (A/HRC/WG.6/1/IDN/1, pár. 2). La Recopilación de la ONU, que 
coincide con la documentación entregada por los Interlocutores, reconoce que Indonesia es "un país multiétnico, multicultural, multirreligioso y multilingüe” (A/HRC/WG.6/1/IDN/2, pár. 37). Sin embargo, se expresa preocupación porque, en la práctica, "se habían puesto en peligro los derechos de los pueblos indígenas ly hace un llamamiento para que] Indonesia garantizara que los conceptos del interés nacional, la modernización y el desarrollo económico y social no se utilizaran como justificación para anular los derechos de los pueblos indígenas" (A/HRC/WG.6/1/IDN/2, pár. 37). Finalmente, el diálogo interactivo vuelve a caracterizarse por dos posiciones diferenciadas (A/HRC/8/23).

En el EPU de Malasia se encuentran plasmados unos argumentos similares a los del EPU de Indonesia, por ejemplo, cuando las autoridades de Malasia enfatizan que como resultado de unos "antecedentes históricos Malasia es en la actualidad étnica y culturalmente diversa” (A/HRC/WG.6/MYS/Rev.1, pár. 5). En el apartado de los derechos culturales, el Informe Nacional afirma que la "representación cultural es importante en un país multicultural como Malasia donde el modo de representación es fundamental para determinar los progresos en materia de integración" (A/HRC/WG.6/4/MYS/1/Rev. 1, pár. 47). Además, declara que la "unidad y la interacción entre las distintas etnias han dado lugar a una sociedad diversa y vibrante que es excepcional en su singularidad" (A/HRC/WG.6/4/MYS/1/Rev.1, pár. 47). Específicamente, afirma que las "tres principales razas no sólo conservan sus respectivas culturas y tradiciones, sino que también son comprensivas y tolerantes y comparten su riqueza cultural" (A/HRC/WG.6/4/MYS/1/Rev.1, pár. 47). Desde el punto de vista de las autoridades de Malasia esa "unidad cultural en la diversidad ha propiciado la coexistencia pacífica y es el principal catalizador de la estabilidad y el crecimiento político de Malasia” (A/HRC/WG.6/4/MYS/1/Rev.1, pár. 47). Hay comentarios análogos sobre la diversidad cultural y racial que se extienden a las referencias a los derechos de los pueblos indígenas, en las que las autoridades declaran que "se alienta a todas las comunidades a hacer 
uso de su acervo cultural y artístico y a darlo a conocer, mejorando así la comprensión entre las culturas y el aprecio por la diversidad cultural” (A/HRC/WG.6/4/MYS/1/Rev.1, pár. 48). Asimismo, el Informe anuncia que la "política cultural de Malasia se basa en su historia, en su situación actual y en el desarrollo al que aspira el país [y] considera que la absorción y la síntesis son los medios necesarios para desarrollar la cultura, fomentar la unidad nacional y reducir la tendencia a la polarización racial" (A/HRC/WG.6/4/MYS/1/Rev.1, pár. 48). Más adelante, declara que el "reto más significativo que afronta Malasia es sacar a los grupos indígenas de su atraso y lograr que se integren socialmente" (A/HRC/WG.6/4/MYS/1/Rev.1, pár. 97). Finalmente, la conclusión declara que "aún y cuando defiende los principios universales de los derechos humanos, Malasia desea recalcar sus propios valores en los derechos humanos que toman en consideración la historia del país y la diversidad religiosa, social y cultural de sus comunidades" (A/HRC/WG.6/4/MYS/1/Rev.1, pár. 114). Malasia afirma que "con esta actitud aspira a mantener y promover el respeto de la armonía social [y que las] prácticas de derechos humanos en Malasia reflejan un sistema de valores asiático más amplio, en el que el bienestar colectivo tiene precedencia sobre los derechos individuales" (A/HRC/WG.6/4/MYS/1/Rev.1, pár. 114).

Por lo que concierne al EPU de la República de Singapur, su Informe Nacional declara que "además del derecho a la protección en condiciones de igualdad, la Constitución contiene disposiciones específicas para la protección de las minorías raciales y religiosas [imponiendo] al Gobierno la responsabilidad de atender a los intereses de las minorías raciales y religiosas" (A/HRC/ WG.6/11/SGP/1, pár. 24). Las autoridades gubernamentales enfatizan que "Singapur suscribe plenamente los principios consagrados en la Declaración Universal de Derechos Humanos” y añaden que respetan el principio de universalidad de los derechos humanos y consideran que los derechos humanos son indivisibles, y que los derechos económicos, sociales y culturales son tan importantes como los derechos civiles y políticos” (A/HRC/WG.6/11/SGP/1, pár. 33). Estas 
declaraciones se completan estableciendo que la "consecución y aplicación de todos los derechos debe, sin embargo, tener en cuenta las circunstancias y aspiraciones nacionales específicas" (A/HRC/ WG.6/11/SGP/1, pár. 33). De modo similar, en el diálogo interactivo Singapur expresó que "todo el mundo debía respetar la normativa universal de los derechos humanos [ya que] la interpretación y la efectividad de los derechos no podía separarse de su contexto social y debían evolucionar al mismo tiempo que la sociedad" (A/HRC/18/11, pár. 7).

\section{El relativismo cultural en el Oriente Medio}

El Informe Nacional de la República de Iraq (A/HRC/ WG.6/7/IRQ/1) no aporta ninguna referencia directa a la cuestión de la universalidad de los derechos humanos o a la incidencia de la diversidad cultural. La única incidencia tangencial se encuentra cuando los Interlocutores manifiestan que "como consecuencia de las tradiciones culturales, se impedía con frecuencia a las niñas que vivían en las zonas rurales proseguir su escolaridad después de los 12 o los 15 años, y que el Ministerio de Educación permanecía mudo e impasible, sin tomar las medidas necesarias para aplicar la Ley de enseñanza obligatoria” (A/HRC/WG.6/7/IRQ/3, pár. 46). También declaran que "la violencia contra las mujeres y las niñas seguía siendo un grave problema y que entre los autores de esa violencia había miembros de los grupos rebeldes y las milicias, soldados y policías" (A/HRC/WG.6/7/IRQ/3, pár. 22). Tanto la Recopilación de la ONU (A/HRC/WG.6/7/IRQ/2) como el Resumen de los Interlocutores reflejan una preocupación similar por la aplicación generalizada de la pena de muerte, por los efectos de la violencia y la inseguridad como consecuencia del estado de guerra, así como por el frecuente quebrantamiento de las reglas de la guerra por parte de los grupos armados. La Recopilación expresa preocupación por unos "grupos armados que seguían ignorando la distinción entre los civiles y los combatientes [con] casos de muertes de civiles causadas por las 
fuerzas de seguridad iraquíes y por las operaciones militares de la fuerza multinacional en el Iraq [junto con] la utilización de mercenarios como medio para violar los derechos humanos [y con ataques] indiscriminados realizados por empleados de las empresas de la seguridad privada, que habían herido o matado a civiles [con] ataques sistemáticos generalizados contra la población civil [que] constituían crímenes de lesa humanidad" (A/HRC/WG.6/7/IRQ/2, pár. 16). Asimismo, como ya pasó con Abu Ghraib, se afirma que en Iraq funcionaban decenas de centros de detención, mayoritariamente secretos y que operan bajo el control de un gobierno extranjero. Además, declara que la implementación de la ley antiterrorista constituye una violación clara de la legislación relativa a los derechos humanos. Por tanto, las prácticas de algunos países occidentales y exponentes del universalismo se pueden cuestionar gravemente, a la vez que deriva en una incidencia respecto a la coherencia de su defensa universalista y contribuye a validar argumentos de los estados que presentan una más firme defensa del relativismo cultural.

En el caso de la República del Yemen, su Informe Nacional declara que "el Gobierno del Yemen considera que el mecanismo de examen periódico universal es un punto de partida para mejorar la situación de los derechos humanos en condiciones de imparcialidad, objetividad y transparencia totales y en el contexto de un diálogo y una cooperación constructivos encaminados al refuerzo y a la protección de los derechos humanos" (A/HRC/WG.6/5/YEM/1, p. 31), sin mencionar la universalidad. A pesar que el Informe Nacional declara que ha establecido un "Consejo Nacional de la Mujer", la Recopilación de la ONU manifiesta una "especial preocupación, entre otras cosas, porque se siguieran produciendo con profusión actos de discriminación contra la mujer y que su desarrollo y promoción no hubieran mejorado de forma significativa, o incluso se hubieran deteriorado con respecto a determinadas cuestiones [aconsejando] que examinara cuidadosamente todas las recomendaciones que le había formulado y se asegurara de que sus obligaciones en virtud de la CEDM, sus principios religiosos y sus valores culturales y sociales 
fueran compatibles, a fin de promover y proteger plenamente los derechos humanos y las libertades fundamentales de las mujeres del Yemen" (A/HRC/WG.6/5/YEM/2, pár. 8). Además, la Recopilación declara que "en 2005, el Comité de Derechos Humanos observó con preocupación que el Yemen justificaba la falta de progresos en varias cuestiones importantes alegando la imposibilidad, a su juicio, de respetar al mismo tiempo los principios religiosos y ciertas obligaciones contraídas en virtud del Pacto Internacional de Derechos Civiles y Políticos [por lo que el] Comité no estuvo de acuerdo con esa interpretación y puso de manifiesto que, en su opinión, se podían tener en cuenta las especificidades culturales y religiosas en el momento de desarrollar los medios adecuados para garantizar el respeto de los derechos humanos universales, pero que ello no podía comprometer el reconocimiento mismo de esos derechos para todos" (A/HRC/WG.6/5/YEM/2, pár. 7). En el diálogo interactivo, aparte de la permanencia de las posturas que se han identificado previamente, cabría subrayar que mientras que Israel “expresó preocupación por los matrimonios precoces forzados, los draconianos métodos de ejecución y de castigo y la discriminación y la violencia sistemáticas contra las mujeres y los niños" (A/HRC/12/13, pár. 21), la República Islámica de Irán “celebró los esfuerzos del Yemen por promover los derechos humanos y afrontar los problemas, teniendo debidamente en cuenta las particularidades nacionales y regionales y el contexto histórico, cultural y religioso" (A/HRC/12/13, pár. 42). Otros países vecinos, como la República Árabe de Siria hicieron "hincapié de los grandes esfuerzos del Yemen por fortalecer los derechos humanos” (A/HRC/12/13, pár. 45).

Con referencia a la República Árabe de Siria, no aparecen menciones explícitas al relativismo cultural en los documentos de su EPU. Las autoridades declararon en su Informe Nacional, preparado durante la guerra civil, que han promulgado un decreto reafirmando la Constitución y protegiendo "la libertad de expresión y el derecho de las personas a acceder a la información de interés público, y también en los derechos fundamentales conforme a los conceptos propios de 
la cultura y los valores de la nación, así como en la responsabilidad que atañe a la información en lo tocante a difundir el conocimiento y servir de cauce para la expresión de los intereses esenciales de la población" (A/HRC/WG.6/12/SYR/1, pár. 91). Por otro lado, referente a la situación de la mujer, el Informe Nacional afirma que el "Estado garantiza a la mujer todas las oportunidades que le brinda la participación efectiva y completa en la vida política, social, cultural y económica" (A/HRC/WG.6/12/SYR/1, P.6). A tal efecto, el Informe reconoce la obligación de monitorizar la implementación de los derechos anunciados en la CEDM. Sin embargo, cabe destacar que las aportaciones relatadas en el Informe respecto a la mujer vuelven a contrastar con lo expuesto en la Recopilación de la ONU, donde se establece que el CEDM "instó a Siria a que abordara las actitudes estereotipadas respecto al rol y las responsabilidades de las mujeres y los hombres, incluidas las pautas y las normas culturales ocultas que perpetuaban la discriminación directa e indirecta contra las mujeres y las niñas" (A/HRC/WG.6/12/SYR/2, pár. 26). Según las autoridades sirias su Informe Nacional "refleja el alcance del compromiso de la República Árabe Siria con el refuerzo y la garantía de los derechos humanos en el marco internacional de los derechos humanos y de conformidad con la Carta de las Naciones Unidas, la Declaración Universal de Derechos Humanos y los tratados internacionales conexos a los que se ha adherido la República Árabe Siria" (A/HRC/WG.6/12/SYR/1, pár. 5). En el diálogo interactivo, mientras los estados occidentales, incluyendo los estados latinoamericanos, se ciñen en los derechos de los que se manifestaban en contra del régimen sirio, la República Islámica de Irán "destacó el establecimiento de diversos mecanismos de derechos humanos, así como logros en los derechos económicos, sociales y culturales" (A/HRC/19/11, pár. 35). Además, de manera destacada, en el diálogo interactivo, Siria declara que "la cultura de los derechos humanos se ha visto afligida por una enfermedad peligrosa, ya que los estados occidentales han actuado como si los derechos humanos fueran un producto cultural que traían a los países en vías de desarrollo" (A/HRC/19/11, pár. 16). La delegación de Siria continuó afirmando 
que "los registros de derechos humanos de algunos estados occidentales son cuanto menos honrosos, en particular cuando han invadido otros países y perseguido una política de 'doble criterio' [con] sanciones en las instituciones internacionales, y en consecuencia usando los derechos humanos como una herramienta para asegurar su hegemonía por encima de otros países en la arena internacional" (A/HRC/19/11, pár. 16).

\section{El relativismo cultural en América Latina y el Caribe}

El Informe Nacional de la República de Colombia declara que el país "avanza en el reto de medir adecuadamente los DESC [los derechos económicos, sociales y culturales] para poder garantizarlos integral y universalmente" (A/HRC/WG.6/3/COL/1, pár. 96). Como en gran parte del EPU de Colombia, en el Informe Nacional se hace mucho eco del conflicto armado interno y del proceso de "Justicia y Paz" que tenía lugar antes de la preparación del Informe. El Informe no contiene más referencias a la universalidad y a la diversidad cultural, aunque pone énfasis en el compromiso de su Constitución y de sus instituciones nacionales con respeto a la democracia y a los derechos humanos, así como hacia el mecanismo del EPU. Finalmente, la delegación declara que las "comunidades indígenas y afrocolombianas cuentan con curules especiales de representación política, sus autoridades son reconocidas como tales, poseen títulos de propiedad colectiva de la tierra y la utilizan según sus costumbres ancestrales, son sujetos de acciones afirmativas en salud, educación y cultura, entre otros y cuentan con espacios de interlocución con el Estado" (A/HRC/WG.6/3/COL/1, pár. 60). En el diálogo interactivo de Colombia, todos los estados destacaron los esfuerzos de este país, aunque también expresaron preocupación respecto a la resolución del conflicto interno y sobre sus consecuencias para la población. A pesar de todo, una vez más se pueden distinguir las posiciones que "recomiendan" de las que "exigen”. 
El Informe Nacional de los Estados Unidos Mexicanos afirma que este país "ha transitado por un proceso de significativas transformaciones en sus instituciones políticas, jurídicas y sociales que, sobre todo a partir de la última década, se han traducido en un mayor goce de todos los derechos humanos en el país" (A/HRC/ WG.6/4/MEX/1, pár. 2). Las autoridades mexicanas afirmaron que se habían comprometido en la implementación de los derechos humanos en sus políticas y en su legislación. Además, declara que el "proceso de transformación en materia de derechos humanos es irreversible [ya que se] ha iniciado un auténtico cambio cultural" (A/HRC/WG.6/4/MEX/1, pár. 3). A pesar de ello, el Informe admite problemas similares a los estados vecinos, tanto en la lucha contra el crimen organizado como hacia la inseguridad pública, así como en cuanto a limitaciones similares en el sistema de justicia y de deficiencias en las condiciones penitenciarias. México también declara que estaba ejecutando medidas especiales para la protección de la libertad de expresión, para garantizar las reparaciones, para poner en práctica medidas para la erradicación de la pobreza y, en especial, gestionando medidas diseñadas para contrarrestar la discriminación por motivos de género y para proteger los menores, las minorías y los migrantes. Finalmente, el Informe Nacional afirma que "México es un país pluricultural con raíces indígenas" (A/HRC/ WG.6/4/MEX/1, pár. 114), y destaca que en "2001 se reformaron diversos artículos de la Constitución a fin de reconocer la composición pluricultural de la Nación y los derechos individuales y colectivos de los pueblos indígenas [incluyendo] el derecho al reconocimiento como pueblo o comunidad indígena, a la auto adscripción, a la libre determinación en un marco constitucional de autonomía que asegure la unidad nacional, a aplicar sus sistemas normativos internos, a la preservación de su identidad cultural, a la tierra que habitan, a la consulta y participación, a acceder plenamente a la jurisdicción del Estado y el derecho al desarrollo" (A/HRC/ WG.6/4/MEX/1, pár. 115). De modo similar, en la presentación de su Informe, México declara que "participa en el mecanismo del EPU porque está convencido de que la promoción y la protección de los 
derechos humanos es una obligación irrenunciable y un imperativo ético universal y que la cooperación con los mecanismos internacionales de derechos humanos es un instrumento inapreciable para favorecer los cambios estructurales internos" (A/HRC/11/27, pár. 5). También reafirma que a "la población indígena le asisten los mismos derechos que a todos los demás miembros de la nación [ya que según] la Constitución mexicana, los pueblos indígenas también gozan de derechos específicos debido a sus diferencias culturales" (A/HRC/11/27, pár. 86). En consecuencia y a tenor de lo expuesto, se puede poner en tela de juicio que México deba de formar parte de un mismo grupo que incluya a otros estados enumerados en la presente.

El Informe Nacional de la República de Cuba declara en su introducción que el "triunfo de la Revolución [...] permitió al pueblo cubano alcanzar su verdadera independencia y establecer las condiciones para el disfrute pleno y universal de todos los derechos humanos [y que las] profundas transformaciones económicas, políticas y sociales emprendidas, hicieron posible la erradicación de las injusticias estructurales heredadas de la dominación colonial y neocolonial que sufrió el país [estableciendo] las bases [y avanzando] de modo permanente en la construcción de una sociedad democrática, justa, participativa, equitativa y solidaria” (A/HRC/ WG.6/4/CUB/1, pár. 3). El Informe defiende un sistema político que se define como democrático y que se encuentra facilitado por unas instituciones que se afirman como abiertas a las reformas. Además, el gobierno declara que defiende los derechos civiles y que ha tomado medidas por lo que refiere a la pena de muerte, las condiciones de encarcelamiento, las garantías respecto a la libertad y a la seguridad individual, la libertad religiosa y la libertad de pensamiento, además de dar un apoyo constante a la educación y la sanidad. Entre las numerosas referencias a una campaña anticubana dirigida por los Estados Unidos, Cuba declara en su Informe que a "pesar de su oposición de principios frente a [...] espurias maniobras, el Gobierno Cubano nunca interrumpió su cooperación con los mecanismos de 
derechos humanos que se aplican de forma universal y sobre bases no discriminatorias" (A/HRC/WG.6/4/CUB/1, pár. 108). Por otro lado, la Recopilación de la ONU detalla las peticiones de la Organización para establecer unos organismos independientes con mandato en los derechos humanos, a la vez que expresa preocupación por la discriminación, la tortura, las condiciones en las prisiones, las detenciones arbitrarias, la violencia doméstica, la prostitución y otras formas de violencia contra las mujeres. Por ejemplo, menciona que "deberían adoptarse medidas para modificar las actitudes determinadas culturalmente que seguían tolerando la violencia contra la mujer" (A/HRC/WG.6/4/CUB/2, pár. 17). De un modo similar, se expresa preocupación por la práctica excesiva de juicios militares y se hace un llamamiento a favor de unos procedimientos legales que se correspondan con la DUDH. Sin embargo, la Recopilación acoge con satisfacción algunas mejoras en los derechos de las mujeres, así como hacia los sistemas de sanidad y educación públicos. Finalmente, comenta que en el año 2006 “Cuba confirmó su voluntad de promover en el Consejo de Derechos Humanos sus iniciativas tradicionales sobre el derecho a la alimentación, la promoción de los derechos culturales y el respeto a la diversidad cultural, y la promoción de la paz para el disfrute de todos los derechos humanos" (A/HRC/WG.6/4/CUB/2, pár. 53). Asimismo, prosigue estableciendo que "Cuba prometió que continuaría trabajando en el desarrollo progresivo de los derechos de tercera generación y en particular, del valor de la solidaridad internacional, así como en la promoción de las iniciativas tradicionales del Movimiento de los Países No Alineados en materia de derechos humanos, y en particular, la relativa al derecho al desarrollo" (A/HRC/ WG.6/4/CUB/2, pár. 53). Al Resumen de los 326 interlocutores (Anexo A/HRC/WG.6/4/CUB/3) y a las aportaciones que se hacen se pone de manifiesto un contraste evidente según el origen de las aportaciones. La denuncia de violaciones de los derechos humanos proviene sobretodo de las organizaciones de derechos humanos internacionales, mientras que las organizaciones oficiales nacionales y los simpatizantes internacionales defienden los esfuerzos del 
régimen respecto a los derechos humanos. Se aprecia una situación similar, cuando en el diálogo interactivo, todos los países asiáticos partidarios del relativismo cultural hacen declaraciones elogiando los esfuerzos de Cuba respecto a los derechos humanos, a pesar de las condiciones impuestas por el embargo, con el apoyo de numerosos pronunciamientos de la parte de los países en vías de desarrollo. Por otro lado, los estados occidentales hacen unas declaraciones que contrastan, con unas recomendaciones y peticiones muy específicas, aunque en ocasiones con ciertos elogios a los esfuerzos realizados. Como pasa en la mayoría de los exámenes mencionados anteriormente, en el diálogo interactivo cubano se pueden identificar claramente dos bloques de países: los que hacen unos comentarios elogiosos y los que condenan y hacen unas peticiones específicas (A/HRC/11/22*).

\section{Conclusiones}

El relativismo cultural contemporáneo se presenta dividido en categorías según los orígenes de sus argumentos. No obstante, estos grupos no son uniformes y ciertos casos pueden combinar distintas formas y distintos grados de intensidad. A lo largo del estudio estos grados de intensidad se encuentran reflejados en unos países que expresan de manera explícita y directa, o de manera implícita e indirecta su respaldo al relativismo cultural aplicado a los derechos humanos. El análisis presentado permite actualizar la lista de países partidarios del relativismo cultural durante la primera y la segunda década del nuevo milenio y, a la vez, permite establecer tres grupos de países.

En primer lugar, podemos identificar una forma más radical de relativismo cultural que se fundamenta en el discurso revolucionario. Este relativismo cultural revolucionario se puede apreciar en unos países con unos regímenes de origen revolucionario, de corte comunista o islamista, que presentan su principal punto de fricción con las normas internacionales de derechos humanos en sus 
reticencias respecto a la libertad política, individual y religiosa. Junto a esta negación del pluralismo, encontramos una frecuente asociación de su argumentación a una oposición respecto a unos grupos de presión colonialistas y, particularmente, un rechazo hacia las injerencias en sus asuntos internos, incluso en el caso que estas interferencias se fundamenten en prevenir los actos represivos, las violaciones de la integridad física, la igualdad de géneros, o la defensa de la libertad ideológica. Dentro del relativismo cultural radical/revolucionario, distinguimos por un lado una forma política basada en la revolución comunista, mientras que, por otro lado, podemos apreciar una forma religiosa fundamentada en la revolución islámica. A tenor de las aportaciones expuestas, podemos determinar que la República Islámica de Irán encabeza el grupo de los países partidarios del relativismo cultural con una aportación extensa, explícita y directa, que se circunscribe dentro del formato del islamismo revolucionario y que, además, se distingue por distintas iniciativas y por la facilitación de un sistema institucional para el relativismo cultural. Por lo que refiere al relativismo cultural de corte comunista y socialista encontramos referencias explícitas y directas de la mano de la República Socialista del Vietnam, la República Popular de la China y la República de Cuba.

Sin embargo, las aportaciones complementarias al EPU, concebidas como una fuente de contraste, detallan unas prácticas preocupantes en estos estados que suponen un quebrantamiento de los derechos humanos individuales más elementales, que además son a menudo sistemáticas y tratadas por el estado en cuestión con unas respuestas pasivas y ambiguas. La información de la ONU y de los Interlocutores revelan que en los países más partidarios del relativismo cultural se dan prácticas que oscilan desde la tortura y las ejecuciones, hasta la censura estructural y la limitación de la libertad de expresión, pasando por la prohibición de "religiones extranjeras de dominio" o de fórmulas políticas disidentes, así como a la discriminación estructural de género, la represión de la libertad sexual y, con todo ello, a menudo implicando una autodeterminación 
colectiva o nacional que limita o impide la autodeterminación individual.

En segundo lugar, distinguimos un grupo de países que se ubicarían en una zona gris, con una argumentación intermedia y poco clara, que tienen sus antecedentes en las revoluciones socialistas, en fundamentos islamistas o en una concepción del estado de tipo multicultural. Estos posicionamientos menos explícitos, expresados por la vía de la omisión de la universalidad en sus contribuciones o con solo posicionamientos en los procesos interactivos, gozan del respaldo de los países con unas posiciones más claras y anteriormente detallados. Este grupo se encuentra conformado por la Unión de Myanmar en el grupo con antecedentes socialistas; la República Islámica del Pakistán, la República del Yemen, y la República Árabe de Siria en el grupo de países fundamentados en una posición islamista; y la República de Indonesia y Malasia en el grupo de países que se basan en la reivindicación multicultural, que conformarían una forma de relativismo cultural moderado fundamentado en unas comunidades diversas.

De modo similar al primer grupo y a tenor de la documentación complementaria, el principal punto de fricción de este segundo grupo con los estándares universales de los derechos humanos residiría en la incidencia de ciertas prácticas tradicionales y religiosas, en la derivada aplicación de las libertades, así como en la conjugación de la autodeterminación colectiva con la autodeterminación individual, además de la autodeterminación de ciertos colectivos, minorías o comunidades indígenas $\mathrm{y}$, todo ello, con la incidencia de los principios propios de los derechos humanos, como es el caso de la igualdad de género o la libertad sexual. En el caso de los países con fundamento islámico, se puede apreciar un particular impacto con los derechos humanos con la aplicación de la ley de la sharia. El conflicto con las normas de la sharia también se puede observar en otras evaluaciones, como la de Mauritania entre numerosos ejemplos en África, Asia y Oriente Medio, pero se utiliza mayoritariamente como argumento a favor del relativismo cultural, directamente en el EPU de 
Irán, e indirectamente en los EPUs de los países islámicos de este grupo. Estas aportaciones indirectas se pueden apreciar tanto en los procesos interactivos del EPU como en la documentación facilitada por el estado, que no solo contienen elementos propios del relativismo cultural, sino que también una reserva generalizada hacia el uso de la terminología universalista.

En tercer lugar, encontramos un grupo de países, que Otto y Cerna habían ubicado como pertenecientes al grupo de los estados partidarios del relativismo cultural y que ahora presentarían cierta tendencia a la adscripción hacia los valores del universalismo. Este grupo está conformado por los Estados Unidos Mexicanos, que presenta el posicionamiento más claro en este sentido, junto con la República de Singapur y la República de Colombia, con posiciones menos claras, y finalmente con el caso añadido de la República de Iraq. Al margen de Iraq, este grupo presenta un posicionamiento particular que proviene de unos países con unas comunidades diversas y que defienden un modelo de diversidad que busca garantizar la autodeterminación individual y colectiva, a la vez que reivindican los valores universales de los derechos humanos sin grandes reservas. Esta posición se puede identificar especialmente en los EPUs de Colombia y México, guardando cierta relación con los posicionamientos de los archipiélagos asiáticos de Indonesia y Malasia, aunque parece presentar un posicionamiento diferente con su aproximación al trato de las comunidades indígenas y en su visión global de los derechos humanos, que en los estados latinoamericanos se fundamenta en la protección de las minorías y de las comunidades indígenas, junto con la facilitación de su autodeterminación y que, a la vez, son principios que se enmarcan dentro de los cauces de las normas internacionales de los derechos humanos.

En todo caso parece que los países que durante el primer ciclo del EPU se encontraban sumergidos en conflictos armados, notablemente Myanmar, Siria, Colombia e Iraq, permanecen influenciados por sus socios partidarios del relativismo cultural en los dos primeros casos (Myanmar y Siria), mientras que los últimos 
(Colombia, y el Iraq pre Estado Islámico) de la parte de los universalistas. El caso de Iraq no parece responder a un cambio genuino, sino que aparenta resultar de la invasión y de la ocupación de la mano de ciertos exponentes occidentales del universalismo que, con ello, reflejaron cierta ambigüedad de su base moral, que materializa un doble discurso y en una teoría y en práctica desdoblada, que erosiona su credibilidad y, a la vez, substancian los temores al colonialismo, invasión y dominio que exponen los países partidarios del relativismo cultural.

Por otra parte, los estados que representan las formas más radicales de relativismo cultural ponen de manifiesto que mientras en el ámbito internacional sus autoridades proclaman la diversidad, son incoherentes en la aplicación de esta diversidad a nivel nacional. Los principales exponentes del relativismo cultural demuestran con sus prácticas y su situación actual que la diversidad, en el ámbito local, es precisamente el elemento que más temen. Consecuentemente, en la escena internacional apelan a la excusa cultural y a la pluralidad, mientras que dentro de sus fronteras adoptan un enfoque represivo respecto al contrato social, con miedo a la oposición o a la disidencia, aunque supuestamente representando a la voluntad del "pueblo" o de "Dios". Por otro lado, en el caso del grupo de estados partidarios de un relativismo cultural comunista o socialista denotan una confusión esencial en la manera como relacionan directamente un régimen político con la dignidad de la vida humana. La dignidad humana individual no necesariamente deriva de una fórmula política colectiva. De la misma manera que, cuando un régimen político producto de una revolución se instala en el poder deja de ser revolucionario.

Podemos concluir que los países que actualmente defienden el relativismo cultural presentan alguna modificación respecto a los estados identificados como partidarios del relativismo cultural en los derechos humanos al finalizar la Guerra Fría. La gran mayoría de los procesos interactivos del EPU revelan que además hay dos grandes grupos diferenciados de países en la comunidad internacional con 
respecto los derechos humanos: un grupo que representa los que condenan, mayoritariamente conformado por estados occidentales y que formulan peticiones y recomendaciones concisas; y otro grupo mayoritario, con los demás estados, a menudo siendo estados en vías de desarrollo, que elogian y recomiendan seguir adelante con los progresos y a diseminar la información. Considerando las estipulaciones del EPU sobre los principios y los objetivos, no es claro de qué manera gestionará tal conflicto el CDH o como se mejorarán los registros de derechos humanos y la protección y el cumplimento de estas normas internacionales con una mayoría evidente de países satisfechos, y muy a pesar de las informaciones que arrojan las fuentes subsidiarias. Asimismo, hay razones para dudar que gran parte de estas evaluaciones cumplan los principios institucionales definidos en la normativa, como por ejemplo el principio de la "no-politización" y el de la "no-confrontación".

Los derechos humanos y el EPU pueden debilitarse si son utilizados como una fuente de enfrentamiento político continuado. El $\mathrm{CDH}$ no debería convertirse en una arena política para presumir superioridad moral, o de reivindicación de agravios históricos, ya que la función del EPU es la mejora y la cooperación en las prácticas de derechos humanos en el presente y futuro e, idealmente, debería constituir el instrumento para poner las bases morales comunes, mundiales y mínimas para asegurar la universalidad y el mínimo común denominador moral, que a la vez podría facilitar el establecimiento de un tribunal internacional de derechos humanos. Finalmente, podemos concluir que todos los estados del EPU tienen buenas y malas prácticas en materia de derechos humanos y que todos aceptan la validez del EPU y comparten la opinio juris de la existencia de los derechos humanos como tal.

A lo mejor, tal y como expuso la Sociedad Americana de Antropología, qué se considera bueno y malo puede variar según la cultura. Sin embargo, la definición central de los derechos humanos no solo se basa en el bien y el mal social en sí mismo, sino en la incidencia de unas formas de violencia individualizada, innecesaria y 
arbitraria. Si los redactores del borrador de la Declaración Universal persistieron en su propósito fue por la convicción de que el sufrimiento humano individual no puede depender de la cultura colectiva. La autodeterminación colectiva siempre debería autorizar la autodeterminación individual. La represión y la violencia nunca pueden ser culturales, ya que la cultura se basa en las ideas, y la práctica de la represión es precisamente el principal recurso a la exterminación de las ideas y de la cultura.

\section{Bibliografía}

American Anthropological Association, Statement on Human Rights of the Executive Board of the: American Anthropologist, New Series, Vol. 49, No. 4, Part 1. (1947).

Ávila R., El relativismo cultural desde la perspectiva de la niñez indígena y la Convención de los Derechos de los Niños. En: Aportes Andinos No.5. Quito: Universidad Andina Simón Bolívar, Sede Ecuador; Programa Andino de Derechos Humanos, marzo 2003. 5 p.

Baderin, Mashood A., International human rights and Islamic law. OUP Oxford, 2003.

Beauchamp T., The compatibility of universal morality, particular moralities, and multiculturalism, Global Bioethics and Human Rights: Contemporary Perspectives (2020).

Boco R. \& Bulanikian G., Derechos humanos: universalismo vs. relativismo cultural. Alteridades 20.40 (2010).

Boco R. \& Bulanikia G., Universalismo vs. relativismo cultural: una batalla por la definición de los derechos humanos y la discriminación. Mediações-Revista de Ciências Sociais 15.1 (2010).

Cerna C., Universality of Human Rights and Cultural Diversity: Implementation of Human Rights in Different Socio-Cultural Contexts, Human Rights Quarterly (1994).

Chimakonam J. \& Agada A., The Sexual Orientation Question in Nigeria: Cultural Relativism Versus Universal Human Rights Concerns. Sexuality \& Culture (2020). 
Declaración de los Ministros y Representativos de los Estados Asiáticos, Declaración de Bangkok, Reunión Regional de Asia para la Conferencia Mundial de Derechos Humanos (2 de abril de 1993).

Donnelly J., Cultural Relativism and Universal Human Rights, Human Rights Quarterly (1984).

Donnelly J., The relative universality of human rights. Human rights Quarterly (2007).

Harvey Brown R. \& Bjawi-Levine L., Cultural Relativism and Universal Human Rights: Contribution from Social Science of the Middle East, The Anthropologist, Volume 4, 2002 - Issue 3.

Johansson Dahre, U., Searching for a middle ground: anthropologists and the debate on the universalism and the cultural relativism of human rights, The International Journal of Human Rights (2017).

Le N. Are Human Rights Universal or Culturally Relative? Peace Review (2016).

Lea D., Human rights as an ideology? Obstacles and benefits. Critical Sociology 46.1 (2020).

Mullender R., Human Rights: Universalism and Cultural Relativism, Critical Review of International Social and Political Philosophy (2003).

Naciones Unidas, Asamblea General, A/HRC/8/23 (14 de mayo de 2008).

—, A/HRC/8/42/Add.1 (25 de agosto de 2008).

—, A/HRC/8/42 (4 de junio de 2008).

—, A/HRC/8/52 (1 de septiembre de 2008).

—, A/HRC/10/29 (9 de noviembre de 2009).

-, A/HRC/10/82 (9 de enero de 2009).

—, A/HRC/10/82/Add.1 (13 de enero de 2009).

—, A/HRC/11/22* Publicado nuevamente por razones técnicas (5 de octubre de 2009).

—, A/HRC/11/25* Publicado nuevamente por razones técnicas (5 de octubre de 2009). 
—, A/HRC/11/27* Publicado nuevamente por razones técnicas (29 de mayo de 2009).

—, A/HRC/11/30* Publicado nuevamente por razones técnicas (5 de octubre de 2009).

—, A/HRC/11/30/Add.1 (3 de junio de 2009).

—, A/HRC/11/37 (16 de octubre de 2009).

—, A/HRC/12/11* Publicado nuevamente por razones técnicas (5 de octubre de 2009).

—, A/HRC/12/11/Add.1 (16 de septiembre de 2009).

—, A/HRC/12/13 (5 de junio de 2009).

—, A/HRC/12/13/Add.1 (23 de septiembre de 2009).

—, A/HRC/12/50 (25 de febrero de 2010).

—, A/HRC/14/12 (15 de marzo de 2010).

—, A/HRC/14/12/Add.1 (3 de junio de 2010).

—, A/HRC/14/14 (15 de marzo de 2010).

—, A/HRC/14/14/Add.1 (8 de junio de 2010).

—, A/HRC/14/37 (23 de octubre de 2012).

—, A/HRC/17/2 (24 de mayo de 2012).

—, A/HRC/17/9 (24 de marzo de 2011).

—, A/HRC/17/9/Add.1 (27 de mayo de 2011).

—, A/HRC/18/11 (11 de julio de 2011).

—, A/HRC/18/11/Add.1 (11 de julio de 2011).

—, A/HRC/18/2 (22 de octubre de 2012).

-, A/HRC/19/11 (24 de enero de 2012).

—, A/HRC/19/2 (24 de mayo de 2013).

—, A/HRC/WG.6/1/IDN/1 (11 de marzo de 2008).

—, A/HRC/WG.6/1/IDN/2 (31 de marzo de 2008).

—, A/HRC/WG.6/1/IDN/3 (6 de marzo de 2008).

—, A/HRC/WG.6/2/PAK/1 (14 de abril de 2008). 
—, A/HRC/WG.6/2/PAK/2 (14 de abril de 2008).

—, A/HRC/WG.6/2/PAK/3 (3 de abril de 2008).

一, A/HRC/WG.6/3/COL/1 (19 de setiembre de 2008).

-, A/HRC/WG.6/3/COL/2 (9 de octubre de 2008).

一, A/HRC/WG.6/3/COL/3 (19 de setiembre de 2008).

-, A/HRC/WG.6/4/CHN/1 (10 de noviembre de 2008).

-, A/HRC/WG.6/4/CHN/2 (16 de diciembre de 2008).

-, A/HRC/WG.6/4/CHN/3 (5 de junio de 2009).

-, A/HRC/WG.6/4/CUB/1 (4 de noviembre de 2008).

-, A/HRC/WG.6/4/CUB/2 (18 de diciembre de 2008).

-, A/HRC/WG.6/4/CUB/3 (28 de diciembre de 2008).

一, A/HRC/WG.6/4/MEX/1 (10 de noviembre de 2008)

-, A/HRC/WG.6/4/MEX/1/Corr.1 (22 de enero de 2009).

一, A/HRC/WG.6/4/MEX/2 (22 de diciembre de 2008).

-, A/HRC/WG.6/4/MEX/3 (19 de diciembre de 2008).

—, A/HRC/WG.6/4/MYS/1/Rev.1 (19 de noviembre de 2008).

-, A/HRC/WG.6/4/MYS/2 (20 de noviembre de 2008).

-, A/HRC/WG.6/4/MYS/3 (27 de octubre de 2008).

-, A/HRC/WG.6/5/VNM/1 (16 de febrero de 2009).

一, A/HRC/WG.6/5/VNM/2 (16 de marzo de 2009).

-, A/HRC/WG.6/5/VNM/3 (23 de febrero de 2009).

-, A/HRC/WG.6/5/YEM/1 (20 de febrero de 2009).

—, A/HRC/WG.6/5/YEM/2 (9 de marzo de 2009).

—, A/HRC/WG.6/5/YEM/3 (19 de febrero de 2009).

-, A/HRC/WG.6/7/IRN/1 (18 de noviembre de 2009).

-, A/HRC/WG.6/7/IRN/2 (25 de noviembre de 2009).

-, A/HRC/WG.6/7/IRN/3 (30 de noviembre de 2009).

—, A/HRC/WG.6/7/IRN/3/Corr.1 (18 de enero de 2010). 
—, A/HRC/WG.6/7/IRQ/1 (18 de enero de 2010).

—, A/HRC/WG.6/7/IRQ/2 (1 de diciembre de 2009).

—, A/HRC/WG.6/7/IRQ/3 (1 de diciembre de 2009).

—, A/HRC/WG.6/10/L.7, Versión avanzada no editada (2 de febrero de 2011).

—, A/HRC/WG.6/10/MMR/1 (10 de noviembre de 2010).

—, A/HRC/WG.6/10/MMR/2 (15 de noviembre de 2010).

—, A/HRC/WG.6/10/MMR/3 (18 de octubre de 2010).

—, A/HRC/WG.6/10/MMR/1/Corr.1 (24 de noviembre de 2010).

—, A/HRC/WG.6/11/SGP/1 (2 de febrero de 2011).

—, A/HRC/WG.6/11/SGP/2 (21 de febrero de 2011).

—, A/HRC/WG.6/11/SGP/3 (21 de febrero de 2011).

—, A/HRC/WG.6/11/SGP/3/Corr.1 (9 de mayo de 2011).

—, A/HRC/WG.6/12/SYR/1 (2 de Setiembre de 2011).

—, A/HRC/WG.6/12/SYR/2 (5 de Setiembre de 2011).

—, A/HRC/WG.6/12/SYR/3 (25 de Julio de 2011).

-, Convención contra la Tortura y Otros Tratos o Penas Crueles, Inhumanos o Degradantes (10 de diciembre de 1984).

-, Convención sobre la Eliminación de todas las formas de

Discriminación contra la Mujer (18 de diciembre de 1979).

-, Convención sobre los Derechos del Niño (20 de noviembre de 1989).

—, Declaración Universal de los Derechos Humanos (8 de diciembre de 1948).

—, Decisión CDH 8/106 (10 de junio de 2008).

—, Decisión CDH 8/124 (12 de junio de 2008).

—, Decisión CDH 10/114 (20 de marzo de 2009).

—, Decisión CDH 11/107 (10 de junio de 2009).

—, Decisión CDH 11/110 (17 de junio de 2009).

—, Decisión CDH 11/113 (11 de junio de 2009). 
—, Decisión CDH 11/116 (12 de junio de 2009).

—, Decisión CDH 12/110 (30 de septiembre de 2009).

—, Decisión CDH 12/112 (30 de septiembre de 2009).

—, Decisión CDH 14/111 (23 de junio de 2010).

—, Decisión CDH 14/113 (23 de junio de 2010).

—, Decisión CDH 18/109 (18 de octubre de 2011).

—, Decisión CDH 19/109 (15 marzo 2012).

—, Resolución 53/208 (14 de enero de 1999).

—, Resolución del Consejo de Derechos Humanos A/RES/60/251 (15 de marzo de 2006).

—, Pacto Internacional de Derechos Civiles y Políticos (16 de diciembre de 1966).

—, Pacto Internacional de Derechos Económicos, Sociales y Culturales (16 de diciembre de 1966).

Naciones Unidas, Carta de las Naciones Unidas (26 de junio de 1945).

Naciones Unidas, Consejo de Derechos Humanos, Resolución 5/1 Construcción institucional del Consejo de Derechos Humanos (18 de junio de 2006).

—, Resolución 8/PRST/1, Modalities and practices for the UPR process (9 de abril de 2008).

-, Resolución PRST/9/2 Follow-up to the President's statement8/1 (24 de septiembre de 2008).

Naciones Unidas, Consejo Económico y Social, Resolución 1996/31 (25 de julio de 1996).

Naciones Unidas, Estatuto de la Corte Internacional de Justicia (26 de junio de 1945).

Naciones Unidas, World Conference on Human Rights, Vienna Declaration and Programme of Action A/CONF.157/23 (12 de julio de 1993).

Otto D., Rethinking the 'Universality' of Human Rights Law, Human Rights Quarterly (1998). 
Prasad A., Cultural Relativism in Human Rights Discourse, Peace Review (2007).

Reichert E., Human Rights: An Examination of Universalism and Cultural Relativism, Journal of Comparative Social Welfare (2006).

Schrijver N., The UN Human Rights Council: A New 'Society of the Committed' or Just Old Wine in New Bottles? Leiden Journal of International Law 20 (2007). 